\title{
Radio Propagation for Wireless Ultra-wide Band Systems
}

\author{
Lijun Li \& Guilin Lu \\ Department of Biological and Chemical Engineering \\ Guangxi University of Technology \\ Liuzhou 545006,China \\ E-mail: lisfishspirit@126.com
}

\begin{abstract}
Wireless ultra-wide band(UWB) wave has been studied by incomplete Mathematical Method, the multiple paths of electromagnetworksic wave on UWB broadband is described from propagate to receive, and analysis mechanism of quality that is affected on the channel by received signal quality: propagation, Teflection, and Transmisson,: practice shows that the Mathematical method can explain the channel characteristics are influenced by the buildings, vegetation and terrain, Review on the crucial effect from the quantum theory on the characteristic and mechanisms of UWB signal, so there are the guidable function which mathematic models are been setted up for the channel indoor and outdoor models .
\end{abstract}

Keywords: Wireless ultra-wide band (UWB), Reflecting, Transmission, Diffraction, Uniform theory of diffraction(UTD)or geometrical theory of diffraction(GTD), Quantum, Quantum entanglement phenomenon

\section{1. preface}

There are many types of Ultra Wide Band (UWB ) signal shape, polycycle pulse shape, Gaussian Doublet pulse shape, etc., for example, two characteristics of Narrow pulse signal pulse :the trans form of signal excitation is single short pulse shape leading edge, and spectrum include regions from direct current to microwave frequency line.

It is physical process that of propagation is electromagnetworksic wave makes for mutual action with medium.

Expression for Model of UWB signal:

$$
p_{x}(t)=\frac{d^{x}(p(t))}{d t^{x}}
$$

We get Power spectral density function:

$$
p(f)=e^{\left(-2(2 \pi f \sigma)^{2}\right.} \int_{-\infty}^{+\infty} \frac{1}{\sqrt{2 \pi} \sigma} \exp \left(\frac{-(t+j 2 \pi f \sigma)^{2}}{2 \sigma^{2}}\right) d t
$$

It compare with pulse types of Gaussian shape :

We have power:

$$
p_{r}(t)=\mathrm{a}_{1}^{2}\left\{e^{-2\left(t-T_{1}\right)^{2} / \tau^{2}}+\left(\frac{a_{2}}{a_{1}}\right)^{2} e^{-2\left(t-T_{2}\right) / \tau^{2}}+2 \frac{\mathrm{a}_{2}}{\mathrm{a}_{1}}\left[\mathrm{e}^{-\left(\mathrm{t}-\mathrm{T}_{1}\right)^{2} / \tau^{2}} e^{-\left(t-T_{2}\right) / \tau^{2}}\right] \operatorname{COS}\left(\phi_{2}-\phi_{1}-\omega \tau\right)\right\}(1-3)^{[2]}
$$

In (1-3), $T_{2}-T_{1}=\tau, a_{1}, a_{2}$ is constant,

It show that Beam is symmetrical, and feeding equalizer filter.

Equivalent circuit:

According to no uniformity properties of microwave, it can Equivalent effect by use networks, in principle:

$\mathrm{p}=\frac{1}{2} \operatorname{Re} \int_{\mathrm{s}}\left(\mathrm{E}_{\mathrm{T}} \times \mathrm{H}_{\mathrm{T}}^{*}\right)$

$$
\mathrm{ds}=\frac{1}{2} \operatorname{Re}\left(\mathrm{UI}^{*}\right)
$$


We get condition of normalizer:

$$
\begin{gathered}
\int_{s}\left(\mathrm{e}_{\mathrm{T}} \times \mathrm{h}_{\mathrm{T}}\right) d s=1 \\
\mathrm{P}(\mathrm{f})=\exp \left(-2(\pi f \sigma)^{2}\right) \int_{-\infty}^{\infty} \frac{1}{\sqrt{2 \pi} \sigma} \exp \left(\frac{-(\mathrm{t}+\mathrm{j} 2 \pi \mathrm{f} \sigma)^{2}}{2 \sigma^{2}}\right) d t
\end{gathered}
$$

It compare with pulse types of Gaussian shape:

$$
\mathrm{P}_{\mathrm{r}}(t)=a_{1}^{2}\left\{e^{-2\left(t-T_{1}\right)^{2} / \tau^{2}}+\left(\frac{a_{2}}{a_{1}}\right)^{2} e^{-2\left(t-T_{2}\right) / \tau^{2}}+2 \frac{\mathrm{a}_{2}}{\mathrm{a}_{1}}\left[\mathrm{e}^{-\left(\mathrm{t}-\mathrm{T}_{1}\right)^{2} / \tau^{2}} \mathrm{e}^{-\left(\mathrm{t}-\mathrm{T}_{2}\right)^{2} / \tau^{2}}\right] \cos \left(\phi_{2}-\phi_{1}-\omega \tau\right)\right\}(1-5)
$$

It shows receiving envelope voltage that it is at different phase shift of two aims.

Network function of UWB signal needn't change into sine wave shape, in general, its spectrum of pulse sequence is made equality by pseudo Random sequence, at most case, it is propagated by code Unit in UWB system.

Considerate parameter and factor of UWB Radio Propagation environment, add electromagnetic properties and electromagnetic properties of mediun and objects, then responsible for electromagnetic wave ,search for properties on Division and frequency

Area, it is primary content.

\section{Propagation mechanisms for UWB Radio}

Frequencies and wavelengths of the electromagnetic spectrum regions for UWB Radio from "3.1 GHz" to " $10.6 \mathrm{GHz}$ ", and from " $\mathrm{C}$ "Band Interleaved to " $\mathrm{X}$ " Band Interleaved.

Around the ideal- free space environment, the research on foundation for UWB Radio Propagation is given by

$\nabla \times E=-\mu \frac{\partial \mathrm{H}}{\partial t}(2-1)$

$\nabla \times \mathrm{H}=\varepsilon \frac{\partial \mathrm{H}}{\partial t}(2-2)$

And average Poynting vector formula:

$\mathrm{S}_{\mathrm{av}}=\frac{1}{2} \mathrm{ReE} \times \mathrm{H}^{*}$

it in lossy homogeneous medium,

If $\sigma, \mu$ and care complex, Equation (2-1), (2-2)

thus becomes

$$
\begin{aligned}
& \nabla \times E=-\mu \frac{\partial \mathrm{H}}{\partial t} \\
& \nabla \times H=\sigma E+\mu \frac{\partial \mathrm{H}}{\partial t}
\end{aligned}
$$

and it in layered media, from the boundary conditions, cindider UWB plane wave incident from the boundary conditions,

Snell's law are given by:

$$
\eta_{1} \sin \theta_{\mathrm{i}}=\eta_{1} \sin \theta_{\mathrm{r}}=\eta_{2} \sin _{\mathrm{t}} \theta_{t}
$$

For free space propation, UWB Radio propagation, the path gain will decrease as the reflected.

In fact, Radio propagation are be effected of loss by medium or barrier, so UWB Radio propagation will received three of different loss: path propagation loss, shadpw fading loss, fast fading loss; and four main effects: shadowy effect, effects of far and wide fading, Multipath effect, Doppler effect.

Base on standard of IEEE .802.15.4a Personal area network(PAN),as fiducial physical layer, wireless USB attest to keep function of wire USB, and offer wireless connect, and testing UWB Devices ,Measurements of the spatial correlation in indoor environment indicate that the multipath arrivals come from a region about the subscriber that around about 10 
metrer, the coherence bandwidth is often taken to be that for which the coherence function, normalized to its peak value, is above 0.5 .

\section{UWB Radio Propagation Procedure}

Single period UWB pulse is nonsinusoidal, there are much difference between period sinusoidal and pulse, also we must consider fully hysteresis loss of absorption wave materials, so Maxwell equations research on transient state solution, not steady state solution to design UWB signal, it can be given modifying Maxwell equations by quote magnetic density.

$$
\begin{aligned}
& \oint H \cdot d l=\int_{s}\left(J+\frac{\partial D}{\partial t}\right) \cdot d s \\
& \nabla \times H=-\frac{\partial B}{\partial t}
\end{aligned}
$$

Method for nonsinusoidal wave Reflection and Transmission in two different nonsinusoidal wave, are the conclusion, that is deal with oscillator, refraction law and snell's law are fitting, where space medium characteristics change slowy with position, electric field strength of field $\mathrm{E}$ are given by Geometrically optical method in uniform mediums, when incident ray encounters of scatter objects near faces, edges, corner, bare wedge-shaped and rolling terrain new diffraction ray.

Diffraction ray don't solve the Boundary problem of electromagnetic field, and don't suit Geometrically optics law, so that distribute for UWB electromagnetic wave field in space is determined by Uniform Theory of diffraction and time dispersion, and determine the receiver electric field strength, the method have been used with success to study 3D complex building environments, and computing the electromagnetic fields have been developed to predict the propagation characteristics.

On the other hand, UTD algorithm is used with every ray by 3D complex radiation field alternating and real condition of all UWB Radio Reflection, Transmission and Diffraction by determine ray azimuth and elevation angle, UTD use Go Geometrical optics principle, for example ,that Ray Tracing technology for ray shooting begin from source of rays, and follow propagating finish propagating simulation, to get UWB channel in pulse response or transfer function with geography information of Arrival.

Several Reflection or Refraction etc., thus that method of UTD can get function of UWB signal voltage

We have studied experiment of UWB wave process from propagation to receive antenna, pulse amplitude and wave traveling have observed, parameter involve normalized pulse funation, spectrum of pseudo random made pulse sequence uniformly.

UWB radio flashy function are gotten during $25 \mathrm{~ns}$ and $1 \mathrm{~ms}$ times, its characteristics are quantum code convolution, and very ability of correct mistaken, so we use quantum theory for analyse its data.

\section{Quantum calculate for UWB Radio propagating}

Significant applications of group theory to quantum physics of crystalline solids derives from the rotational-reflectional symmetry of the entire crystal lattice under a group of rotations, reflections, and translation.

Under line Axiom does not require the commutative law,

$\forall a, b \in G, a \circ b=b \circ a$

When we discuss propagation, we are dealing with microwave radiation theorem, and use five hypothesis of quantum mechanics, its foundation is the solutions of the homogeneous Helmholtz equation

$$
\left(\nabla^{2}+k^{2}\right) \psi(r)=0
$$

And the schrodinger equation

Thus, equation is given by

$$
H \psi(r)=E \psi(r)
$$

Discrete quantum system is an element of $c^{n}$ such that the normalization condition[2].

$$
\sum_{i=1}^{n}\left|\psi^{i}\right|^{2}=1
$$

Is obeyed:

Quantum codes have shown ability strongly in UWB system, and Quantum cryptography Based on entanglement 
phenomenon can change into NP mathe matical problem,-----etc. so its have come true.

According to Quantum no-cloning, quantum position of unknown status travel by quantum entanglement phenomenon.

\section{5. summary}

Computer codes the directions of the rays at both ends of the links for computing the electromagnetic fields have been developed to predit the propagation characteristics for the UWB system simulations, classical is used to analys UWB Radio reflection and Transmission, but diffraction by Edges and corners can only use UTD theory ,and scatter and Radiation need use quantum theory, the simulation results show UWB Radio analysis correctly.

\section{References}

Fawway T.Ulaby Richard K.Moore Adrian K.Fung(1987), MICROWAVE REMOTE SENSING, Volume II Radar Remote Sensing and Surface Scattering and Emission Theory, Addison-wesley publishing company, 1982, pp. 38.

unruh W G.Maintaining Coherence in Quantum Computers. Phys. Rev. A, 51, 992, 1995. 